\title{
EFEITO DO MANEJO DA ENTRELINHA DA SERINGUEIRA SOBRE AS PROPRIEDADES QUÍMICAS DO SOLO, O ESTADO NUTRICIONAL E O CRESCIMENTO ${ }^{1}$
}

\author{
Maria Aparecida Pessôa da Cruz Centurion², José Frederico Centurion ${ }^{3}$, Cassiano Garcia Roque ${ }^{4}$, Renato \\ de Mello Prado ${ }^{5}$ e William Natale ${ }^{3}$
}

\begin{abstract}
RESUMO - O trabalho teve por objetivo analisar a influência do manejo do solo da entrelinha da cultura da seringueira (Hevea brasiliensis) sobre as propriedades químicas do solo, o teor de nutrientes e o crescimento da seringueira. Para isso, implantou-se a seringueira (clones PB 235 e RRIM 701) em Latossolo VermelhoEscuro, em 1992, no Município de Jaboticabal, SP. Os tratamentos consistiram do manejo da entrelinha com: grade, roçadeira e cultura de cobertura utilizando uma leguminosa kudzu tropical (Pueraria phaseoloides). Após oito anos, foram analisadas algumas propriedades químicas do solo (pH, M.O., $\mathrm{H}+\mathrm{Al}, \mathrm{Ca}^{+2}, \mathrm{Mg}^{+2}, \mathrm{~K}^{+}$ e P) na entrelinha da cultura, bem como o teor de macro e micronutrientes e o crescimento da cultura em circunferência à altura do peito. Os diferentes manejos da entrelinha da cultura da seringueira afetaram todos os atributos químicos do solo da camada superficial ( $0-20 \mathrm{~cm}$ de profundidade) analisados. O manejo da entrelinha da seringueira com roçadeira mostrou-se importante no sistema solo-planta. A influência do manejo da entrelinha da seringueira na sua nutrição depende do clone utilizado no sistema de produção.
\end{abstract}

Palavras-chave: Hevea brasiliensis, cultura intercalar, roçadeira, grade e nutrição.

\section{EFFECT OF INTER ROW MANAGEMENT ON CHEMICAL PROPERTIES OF THE SOIL, NUTRITIONAL STATUS AND DEVELOPMENT OF RUBBER TREE}

\begin{abstract}
- the objective of the present study was to assess the effect of rubber tree inter row management on chemical properties of the soil, nutrient concentrations and development of rubber trees. Rubber trees (PB 235 and 701) were planted on red latossol, in 1992 - Jaboticabal-SP. The treatments consisted of managing rows with harrow, mowing and tropical kudzu (Pueraria phaseoloides) cover crop. After eight years, some chemical properties of the soil were analyzed ( $\mathrm{pH}, \mathrm{O} . \mathrm{M} ., \mathrm{H}+\mathrm{Al}, \mathrm{Ca}^{+2}, \mathrm{Mg}^{+2}, \mathrm{~K}^{+}$and $\mathrm{P}$ ) between rows, the tree nutrient concentrations as well as diameter at breast height were assessed. The different management types between rows affected all the chemical attributes of soil only in the superficial layer ( $0-20 \mathrm{~cm}$ depth) analyzed. The management between rows with mowing was found to be important for the soil-tree system. The influence of the management between rows on tree nutrition depends on the clone used for the production system.
\end{abstract}

Key-words: Hevea brasiliensis, inter-crop system, mowing, harrow, nutrition.

\footnotetext{
${ }^{1}$ Recebido em 06.11.2003 e aceito para publicação em 25.11.2004.

${ }^{2}$ Departamento de Produção Vegetal, Faculdade de Ciências Agrárias e Veterinárias, FCAV/UNESP. Via de Acesso Paulo Donato Castellane, s/n. 14870-000, Jaboticabal-SP.

${ }^{3}$ Departamento de Solos e Adubos, FCAV/Unesp. E-mail: <jfcentur@fcav.unesp.br>.

${ }^{4}$ Programa de Pós-Graduação em Produção Vegetal da FCAV/Unesp.

${ }^{5}$ Departamento de Solos e Adubos, FCAV/Unesp. E-mail: <rmprado@ fcav.unesp.br>.
} 


\section{INTRODUÇÃO}

Os plantios comerciais do gênero Hevea ocupam uma faixa ampla, desde $23^{\circ} \mathrm{N}$ (China) até $25^{\circ} \mathrm{S}$ (São Paulo). Apesar de o Brasil ser o centro de origem da Hevea, o país continua sendo grande importador de borracha natural. Relatórios recentes indicam que em 1997 foram importadas cerca de 67 mil toneladas, para um consumo nacional estimado em 102 mil toneladas e uma produção de 31 mil toneladas de borracha seca (SERINGUEIRA, 1999). Esses dados evidenciam a necessidade de se intensificar o plantio desta cultura, a fim de atender à demanda interna de borracha.

No Estado de São Paulo, o plantio de Hevea atinge uma área plantada de 45 mil ha (CORTEZ, 1998). OPlanalto Paulista está localizado na região considerada preferencial, no país, para o cultivo da seringueira, uma vez que as condições térmicas e hídricas são satisfatórias (ORTOLANI et al., 1983). Além disso, os solos (Latossolos e Argissolos) são aptos ao desenvolvimento e produção da seringueira, visto que não apresentam limitações quanto às propriedades físicas (profundidade efetiva, textura e drenagem interna) e químicas (CENTURION et al., 1995). Some-se a isso o fato de as condições climáticas dessa região não serem favoráveis à ocorrência do mal-das-folhas (Microcyclus ulei - P. Henn.) em níveis epidêmicos, doença fortemente limitante à exploração da cultura em regiões quentes e úmidas.

Conforme Compagnon (1986), em seringais que na fase imatura receberam fertilizante suficiente podese suspender a adubação por quatro anos após o início da sangria, fazendo apenas a aplicação de $\mathrm{N}$, a fim de repor as perdas com em extração do látex. Nesse caso, os níveis de nutrientes seriam monitorados a intervalos de três a cinco anos. Entretanto, os diferentes manejos da entrelinha da seringueira podem alterar a dinâmica de nutrientes no sistema solo-planta, implicando ajustes nos programas de adubação.

O manejo convencional do solo na entrelinha da seringueira utiliza-se de grade, e recentemente têm sido recomendados a roçadora e o plantio de culturas de cobertura. O uso do kudzu-tropical (Pueraria phaseoloides Benth.) como cobertura vegetal na entrelinha dos seringais visa à supressão das capinas e à conservação da umidade e melhoria da fertilidade do solo (CARDOSO, 1989). Além disso, as plantas de cobertura reduzem a intensidade dos processos erosivos do solo (SOUZA, 1996) e, conseqüentemente, as perdas de água e de nutrientes.

Os efeitos benéficos da adubação verde sobre a fertilidade do solo devem-se ao aumento do teor de matéria orgânica, à maior disponibilidade de nutrientes, à melhoria da capacidade de troca de cátions efetiva, à diminuição dos teores de Al-trocável e ao incremento da capacidade de reciclagem e mobilização de nutrientes lixiviados ou pouco solúveis que estejam nas camadas mais profundas do solo (TESTA et al., 1992). No entanto, existem evidências de falta de resposta dos adubos verdes na melhoria da fertilidade do solo (CACERES, 1994). Cabe salientar que os efeitos promovidos pela adubação verde nas propriedades químicas do solo são bastante variáveis, dependendo de fatores como a espécie utilizada, o manejo dado à biomassa, o tempo de permanência dos resíduos no solo, o sistema de produção adotado, as condições edafoclimáticas do local e as interações entre esses fatores.

Em função da importância da Hevea brasiliensis no Brasil, tornam-se necessários adotar sistemas de produção que permitam explorar o potencial dessa espécie no Planalto Paulista. Nesse sentido, é necessária a realização de trabalhos de longa duração com manejo da seringueira em áreas tradicionais de cultivo.

Nesse contexto, o presente trabalho teve por objetivo avaliar a influência do manejo da entrelinha de dois clones de seringueira (PB 235 e RRIM 701), de forma mecânica, com grade, roçadora e com cultura de cobertura, durante oito anos de cultivo, sobre as propriedades químicas do solo e a nutrição e crescimento das plantas de seringueira.

\section{MATERIAL E MÉTODOS}

Oexperimento foi conduzido na Fazenda Experimental da Faculdade de Ciências Agrárias e Veterinárias UNESP, Campus de Jaboticabal, com latitude de $21^{\circ}$ $15^{\prime}$, longitude de $48^{\circ} 15^{\prime}$ e altitude de $595 \mathrm{~m}$. O clima é do tipo Cwa, com verão chuvoso e inverno seco. A seringueira foi implantada em 1992 e conduzida durante oito anos num Latossolo Vermelho-Escuro, textura argilosa (ANDRIOLI e CENTURION, 1999), cujas propriedades químicas estão apresentadas na Tabela 1.

Os clones de seringueira utilizados na área experimental foram o PB 235 (híbrido resultante do cruzamento dos clones primários PB 5/51 x PB 5/72, 
originário da Malásia) e o RRIM 701 (resultante do cruzamento dos clones 44/53 com o RRIM 501). O experimento foi instalado em blocos ao acaso, com quatro repetições, sendo a parcela experimental constituída por 28 plantas (4 linhas com 7 plantas), espaçamento $7,5 \times 2,5 \mathrm{~m}$, sendo as 10 plantas centrais consideradas como parcela útil, perfazendo uma área útil de $175 \mathrm{~m}^{2}$. O plantio foi realizado através de mudas enxertadas.

Os tratamentos foram os seguintes: gradagem, roçadora e cultura de cobertura kudzu tropical (Pueraria phaseoloides). A gradagem ocorreu duas vezes por ano (período chuvoso), utilizando-se a grade aradora 14 x 28 ", tracionada por um trator de pneu ( $82 \mathrm{cv}$.), com profundidade média estimada de atuação de 10$15 \mathrm{~cm}$. A roçadora foi utilizada três vezes por ano (período chuvoso), triturando-se o material vegetal e mantendoo na entrelinha da cultura. Já o kudzu foi semeado a lanço na área e mantido com uma roçada por ano, visando apenas evitar o hábito de trepadeira dessa leguminosa nas árvores de seringueira, uma vez que poderia dificultar seu crescimento. O manejo das plantas daninhas na linha foi efetuado através de aplicação de herbicidas de contato, já os demais tratos culturais, como controle de pragas, doenças e outros, foram de acordo com a necessidade de controle. A adubação foi realizada de acordo com a análise de solo, segundo Raij e Quaggio (1983).

Após oito anos de cultivo, em 2000 os efeitos do manejo na entrelinha da seringueira foram avaliados no solo, no estado nutricional e no desenvolvimento da seringueira. No solo, as propriedades químicas analisadas foram: pH, M.O. (matéria orgânica), H+Al, $\mathrm{Ca}^{+2}, \mathrm{Mg}^{+2}, \mathrm{~K}^{+}$e P. Com esses dados foi calculado a soma de bases, a capacidade de troca catiônica e a saturação por bases, seguindo a metodologia de Raij et al. (2001). Essas amostras de solo foram coletadas na entrelinha da seringueira em quatro profundidades
(0-20, 20-40, 40-60 e 60-100 cm). Na planta, coletaramse folhas de acordo com os procedimentos de Chapman (1966), avaliando os teores foliares de macronutrientes e micronutrientes, conforme a metodologia de Bataglia et al. (1983). O desenvolvimento da seringueira foi avaliado através da circunferência à altura do peito (medida a 1,30 $\mathrm{m}$ de altura do solo).

O estudo estatístico constou da análise de variância, considerando-se para as propriedades químicas do solo três manejos e quatro profundidades e para os nutrientes foliares, três manejos e clones, ambos com quatro repetições. Utilizou-se o teste de Tukey $(\mathrm{P}<0,05)$ para a comparação entre as médias obtidas (PIMENTELGOMES, 1985). As análises foram processadas por meio do Statistical Analysis System (SAS, 1996).

\section{RESULTADOS E DISCUSSÃO}

\subsection{Efeito dos tratamentos nos atributos químicos do solo}

A análise dos resultados indicou diferença significativa entre os tratamentos quanto às variáveis químicas $\mathrm{pH}, \mathrm{H}+\mathrm{Al}, \mathrm{Ca}, \mathrm{Mg}, \mathrm{Ke} \mathrm{P}$ (Tabela 2). Evidenciou, ainda, diferenças significativas entre as profundidades, sendo os maiores valores observados na camada superficial (0-20 cm). Para o $\mathrm{pH}$, maiores valores ocorreram na camada de 60-100. De maneira geral, constatou-se que os manejos afetam, principalmente, a camada superficial do solo.

Entre os manejos da entrelinha houve diferença significativa na camada superficial, com maior $\mathrm{pH}$ e menor concentração de $\mathrm{H}+\mathrm{Al}$ no solo, cultivado com Pueraria e manejado com uso da roçadora. A causa provável disso é que os resíduos deixados na superfície do solo liberam agentes quelantes que complexam o alumínio trocável do solo (HOYT, 1977). Entretanto, esses resultados contrariam Haynes (1986), que afirmou que resíduos vegetais na superfície acidificam o solo.

Tabela 1 - Propriedades químicas do solo da área experimental Table 1 -Chemical properties of the soil from the experimental area

\begin{tabular}{|c|c|c|c|c|c|c|c|c|c|c|c|}
\hline Camada & $\begin{array}{c}\mathrm{pH} \\
\mathrm{KCl}\end{array}$ & M.O. & $\begin{array}{c}\mathrm{P} \\
\text { Resina }\end{array}$ & $\mathrm{K}$ & $\mathrm{Ca}$ & $\mathrm{Mg}$ & $(\mathrm{H}+\mathrm{Al})$ & SB & $\mathrm{T}$ & $\mathrm{Al}$ & $\mathrm{V}$ \\
\hline $\mathrm{cm}$ & & $\mathrm{g} \mathrm{dm}^{-3}$ & $\mathrm{mg} \mathrm{dm}^{-3}$ & \multicolumn{5}{|c|}{$\mathrm{mmol}_{\mathrm{c}} \mathrm{dm}^{-3}$} & & $\%$ & \\
\hline $0-15$ & 6,2 & 23 & 36 & 2,9 & 55 & 19 & 25 & 76,9 & 101,9 & 0,0 & 75 \\
\hline $15-43$ & 4,9 & 14 & 5 & 0,6 & 22 & 11 & 38 & 33,6 & 71,6 & 0,0 & 47 \\
\hline
\end{tabular}


Tabela 2 - Variáveis químicas do solo em função dos manejos mecânicos (grade e roçadeira) e cultura de cobertura (Pueraria), na entrelinha da seringueira após oito anos de cultivo. Jaboticabal, SP

Table 2 - Soil chemical properties as a function of mechanical management (grazer and harrow) and cover crop (Pueraria), in inter rows of rubber trees after eight years of cultivation. Jaboticabal, SP

\begin{tabular}{|c|c|c|c|c|}
\hline \multirow[b]{2}{*}{ Profundidade $(\mathrm{cm})$} & \multicolumn{4}{|c|}{$\mathrm{pH}\left(\mathrm{CaCl}_{2}\right)$} \\
\hline & Grade & Roçadora & Pueraria & Médias \\
\hline $0-20$ & $5,2 \mathrm{Bb}$ & $5,4 \mathrm{ABa}$ & $5,4 \mathrm{Aa}$ & $5,3 \mathrm{~B}$ \\
\hline $20-40$ & $4,9 \mathrm{Ca}$ & $5,1 \mathrm{Ca}$ & $5,0 \mathrm{Ba}$ & $5,0 \mathrm{D}$ \\
\hline $40-60$ & $5,1 \mathrm{BCa}$ & $5,1 \mathrm{BCa}$ & $5,1 \mathrm{Ba}$ & $5,1 \mathrm{C}$ \\
\hline $60-100$ & $5,5 \mathrm{Aa}$ & $5,5 \mathrm{Aa}$ & $5,6 \mathrm{Aa}$ & $5,5 \mathrm{~A}$ \\
\hline \multirow[t]{2}{*}{ Média } & $5,2 \mathrm{a}$ & $5,25 \mathrm{a}$ & $5,27 \mathrm{a}$ & - \\
\hline & \multicolumn{4}{|c|}{$\mathrm{H}+\mathrm{Al}\left(\mathrm{mmol}_{\mathrm{c}} \mathrm{dm}^{-3}\right)$} \\
\hline $0-20$ & $32,5 \mathrm{Aa}$ & $28,8 \mathrm{Ab}$ & $29,5 \mathrm{Ab}$ & $30,3 \mathrm{~A}$ \\
\hline $20-40$ & $31,0 \mathrm{Aa}$ & $28,0 \mathrm{Aa}$ & $30,3 \mathrm{Aa}$ & $29,8 \mathrm{~A}$ \\
\hline $40-60$ & $25,8 \mathrm{Ba}$ & $23,5 \mathrm{Ba}$ & $24,3 \mathrm{Ba}$ & $24,5 \mathrm{~B}$ \\
\hline $60-100$ & $20,5 \mathrm{Ca}$ & $21,5 \mathrm{Ca}$ & $20,5 \mathrm{Ca}$ & $21,8 \mathrm{C}$ \\
\hline \multirow[t]{2}{*}{ Média } & $27,4 \mathrm{a}$ & $25,4 \mathrm{a}$ & $26,1 \mathrm{a}$ & \\
\hline & \multicolumn{4}{|c|}{$\mathrm{Ca}\left(\mathrm{mmol}_{\mathrm{c}} \mathrm{dm}^{-3}\right)$} \\
\hline $0-20$ & $23,0 \mathrm{Ab}$ & $26,8 \mathrm{Ab}$ & $32,5 \mathrm{Aa}$ & $27,4 \mathrm{~A}$ \\
\hline $20-40$ & $16,5 \mathrm{Ba}$ & $17,5 \mathrm{Ba}$ & $19,8 \mathrm{Ba}$ & $17,9 \mathrm{~B}$ \\
\hline $40-60$ & $17,0 \mathrm{Ba}$ & $15,3 \mathrm{Ba}$ & $15,8 \mathrm{Ba}$ & $16,0 \mathrm{BC}$ \\
\hline $60-100$ & $14,0 \mathrm{Ba}$ & $14,5 \mathrm{Ba}$ & $15,5 \mathrm{Ba}$ & $14,7 \mathrm{C}$ \\
\hline \multirow[t]{2}{*}{ Média } & $17,6 \mathrm{a}$ & $18,5 \mathrm{a}$ & $20,9 \mathrm{a}$ & - \\
\hline & \multicolumn{4}{|c|}{$\mathrm{Mg}\left(\mathrm{mmol}_{\mathrm{c}} \mathrm{dm}^{-3}\right)$} \\
\hline $0-20$ & $13,5 \mathrm{Aa}$ & $20,2 \mathrm{Aa}$ & $20,3 \mathrm{Aa}$ & $18,0 \mathrm{~A}$ \\
\hline $20-40$ & $10,8 \mathrm{Aa}$ & $12,3 \mathrm{ABa}$ & $13,8 \mathrm{Aba}$ & $12,3 \mathrm{~B}$ \\
\hline $40-60$ & $10,5 \mathrm{Aa}$ & $9,8 \mathrm{Ba}$ & $9,8 \mathrm{Ba}$ & $10,0 \mathrm{BC}$ \\
\hline 60-100 & 6,3 Aa & $6,5 \mathrm{Ba}$ & $7,0 \mathrm{Ba}$ & $6,6 \mathrm{C}$ \\
\hline \multirow[t]{2}{*}{ Média } & $10,3 \mathrm{c}$ & $12,2 \mathrm{~b}$ & $12,7 \mathrm{a}$ & \\
\hline & \multicolumn{4}{|c|}{$\mathrm{K}\left(\mathrm{mmol}_{\mathrm{c}} \mathrm{dm}^{-3}\right)$} \\
\hline $0-20$ & $2,1 \mathrm{Ab}$ & $2,3 \mathrm{Ab}$ & $2,7 \mathrm{Aa}$ & $2,4 \mathrm{~A}$ \\
\hline $20-40$ & $1,3 \mathrm{Ba}$ & $1,3 \mathrm{Ba}$ & $1,7 \mathrm{Ba}$ & $1,4 \mathrm{~B}$ \\
\hline $40-60$ & $0,9 \mathrm{Ca}$ & $1,1 \mathrm{Ba}$ & $1,1 \mathrm{Ca}$ & $1,0 \mathrm{C}$ \\
\hline 60-100 & $0,5 \mathrm{Cb}$ & $0,5 \mathrm{Cab}$ & $0,6 \mathrm{Da}$ & $0,5 \mathrm{D}$ \\
\hline \multirow[t]{2}{*}{ Média } & $1,2 \mathrm{a}$ & $1,3 \mathrm{a}$ & $1,5 \mathrm{a}$ & - \\
\hline & \multicolumn{4}{|c|}{$\mathrm{P}\left(\mathrm{mg} \mathrm{dm}^{-3}\right)$} \\
\hline $0-20$ & $18,0 \mathrm{Aa}$ & 19,3 Аа & $20,5 \mathrm{Aa}$ & $19,3 \mathrm{~A}$ \\
\hline $20-40$ & $7,5 \mathrm{Ba}$ & $6,8 \mathrm{Ba}$ & 7,3 $\mathrm{Ba}$ & 7,2 B \\
\hline $40-60$ & $6,3 \mathrm{Ba}$ & $5,8 \mathrm{Ba}$ & $5,5 \mathrm{Ba}$ & $5,8 \mathrm{BC}$ \\
\hline $60-100$ & $4,0 \mathrm{Ba}$ & $3,2 \mathrm{Ba}$ & $4,5 \mathrm{Ba}$ & $3,9 \mathrm{C}$ \\
\hline Média & $8,9 \mathrm{a}$ & $8,8 \mathrm{a}$ & $9,4 \mathrm{a}$ & - \\
\hline
\end{tabular}

Médias seguidas de mesma letra não diferem entre si pelo teste de Tukey $(\mathrm{p}<0,05)$, e as letras minúsculas comparam os manejos dentro da mesma profundidade e as maiúsculas, as profundidades dentro de cada tipo de manejo.

Means followed by the same letters did not differ from one another by the Tukey test $(p<0.05)$. Lower case letters compare the types of management within the same depth and capital letters compare the depths within each type of management.

As concentrações de potássio e cálcio no solo com Pueraria foram superiores, dentre os manejos avaliados. Esse efeito da Pueraria no aumento da concentração desses elementos no solo da camada superficial deveu-se à ciclagem de nutrientes, e, segundo Tiwari et al. (1980), a decomposição da matéria orgânica pode ter efeito solubilizante no K nativo do solo, aumentando sua disponibilidade. O manejo com Pueraria aumentou significativamente a concentração de $\mathrm{Mg}$ do solo, porém a concentração de P no solo não foi influenciada pelos manejos (Tabela 2).

O cultivo da Pueraria na entrelinha da seringueira permitiu aumento significativo do teor de matéria orgânica, acompanhado da soma de bases e, conseqüentemente, 
da CTC, e da saturação por bases (Tabela 3). Tal fato é explicado por Testa et al. (1992), pois as leguminosas são capazes de produzir altas quantidades de resíduos, permitindo, assim, redução da lixiviação de cátions e aumento da CTC e da soma de bases do solo. O sistema de cultivo do solo com leguminosas, além de aumentar o teor de matéria orgânica do solo, resulta em diminuição da emissão de $\mathrm{CO}_{2}$ para a atmosfera (BAYER et al., 2000), com benefícios ao meio ambiente. O uso da grade na entrelinha da seringueira, porém, apresentou o menor teor de matéria orgânica na camada superficial do solo. O sistema de cultivo com o uso de grades afeta os estoques de matéria orgânica do solo, pois promove degradação da sua estrutura (PRADO e CENTURION,
2001), levando a intensas perdas de matéria orgânica por mineralização e erosão (CASTRO FILHO et al., 1991). Essa maior degradação da MO na camada superficial do solo, com o uso da grade, pode explicar a menor concentração de bases nessa camada ( $\mathrm{Ca}, \mathrm{Mg}$ e $\mathrm{K}$ ) (Tabela 2).

\subsection{Efeito dos tratamentos no estado nutricional da seringueira}

A análise dos resultados indicou diferença significativa entre os manejos da entrelinha da seringueira sobre os teores foliar de nitrogênio, fósforo e cálcio e, entre os clones, sobre nitrogênio, fósforo, magnésio e boro foliar (Tabela 4).

Tabela 3 - Teor de matéria orgânica, valor de soma de bases, capacidade de troca catiônica e saturação por bases do solo, em função dos manejos mecânicos (grade e roçadeira) e cultura de cobertura (Pueraria), na entrelinha da seringueira após oito anos de cultivo, Jaboticabal, SP

Table 3 - Organic matter content, base sum value, cation exchange capacity and base saturation of soil as a function of the mechanical management (grazer and harrow) and cover crop (Pueraria), in inter rows of rubber trees after eight years of culture, Jaboticabal, SP

\begin{tabular}{|c|c|c|c|c|}
\hline \multirow[b]{2}{*}{ Profundidade $(\mathrm{cm})$} & \multicolumn{4}{|c|}{ Matéria Orgânica $\left(\mathrm{g} \mathrm{kg}^{-1}\right)$} \\
\hline & Grade & Roçadora & Pueraria & Médias \\
\hline $0-20$ & $20,0 \mathrm{Ab}$ & $21,0 \mathrm{Ab}$ & $22,5 \mathrm{Aa}$ & $21,2 \mathrm{~A}$ \\
\hline $20-40$ & $13,3 \mathrm{Ba}$ & $13,5 \mathrm{Ba}$ & $15,0 \mathrm{Ba}$ & $13,9 \mathrm{~B}$ \\
\hline $40-60$ & $12,0 \mathrm{Ba}$ & $10,8 \mathrm{Ca}$ & $11,5 \mathrm{Ca}$ & $11,4 \mathrm{C}$ \\
\hline 60-100 & $9,3 \mathrm{Ca}$ & $8,5 \mathrm{Ca}$ & $9,5 \mathrm{Ca}$ & $9,1 \mathrm{D}$ \\
\hline \multirow[t]{2}{*}{ Média } & $13,6 \mathrm{ab}$ & $13,4 \mathrm{~b}$ & $14,625 \mathrm{a}$ & - \\
\hline & \multicolumn{4}{|c|}{ Soma de Bases $\left(\mathrm{mmol}_{\mathrm{c}} \mathrm{dm}^{-3}\right)$} \\
\hline $0-20$ & $38,6 \mathrm{Ab}$ & $49,3 \mathrm{Aab}$ & $55,5 \mathrm{Aa}$ & $47,8 \mathrm{~A}$ \\
\hline $20-40$ & $28,5 \mathrm{Ba}$ & $31,1 \mathrm{Ba}$ & $35,2 \mathrm{Ba}$ & $31,6 \mathrm{~B}$ \\
\hline $40-60$ & $28,4 \mathrm{Ba}$ & $26,1 \mathrm{Ba}$ & $26,6 \mathrm{Ba}$ & $27,0 \mathrm{BC}$ \\
\hline$\underline{60-100}$ & $20,7 \mathrm{Ba}$ & $21,5 \mathrm{Ba}$ & $23,1 \mathrm{Ba}$ & $21,8 \mathrm{C}$ \\
\hline \multirow[t]{2}{*}{ Média } & 29,1 a & 32,0 a & $35,1 \mathrm{a}$ & - \\
\hline & \multicolumn{4}{|c|}{ Capacidade de Troca Catiônica $\left(\mathrm{mmol}_{\mathrm{c}} \mathrm{dm}^{-3}\right)$} \\
\hline $0-20$ & $71,1 \mathrm{Aa}$ & $78,0 \mathrm{Aa}$ & $85,0 \mathrm{Aa}$ & $78,0 \mathrm{~A}$ \\
\hline $20-40$ & $59,5 \mathrm{Ba}$ & $58,8 \mathrm{Ba}$ & $65,5 \mathrm{Ba}$ & $61,3 \mathrm{~B}$ \\
\hline $40-60$ & $54,1 \mathrm{Ba}$ & $49,6 \mathrm{BCa}$ & $50,8 \mathrm{BCa}$ & $51,5 \mathrm{C}$ \\
\hline $60-100$ & $41,2 \mathrm{Ca}$ & $43,0 \mathrm{Ca}$ & $43,6 \mathrm{Ca}$ & $42,6 \mathrm{D}$ \\
\hline \multirow[t]{2}{*}{ Média } & 56,5 a & 57,4 a & $61,2 \mathrm{a}$ & - \\
\hline & \multicolumn{4}{|c|}{ Saturação por Bases $(\%)$} \\
\hline $0-20$ & $54,0 \mathrm{Ab}$ & $62,5 \mathrm{Aa}$ & $64,8 \mathrm{Aa}$ & $60,4 \mathrm{~A}$ \\
\hline $20-40$ & $47,8 \mathrm{Aa}$ & $52,5 \mathrm{ABa}$ & $53,5 \mathrm{Ba}$ & $51,3 \mathrm{~B}$ \\
\hline $40-60$ & $52,5 \mathrm{Aa}$ & $52,5 \mathrm{ABa}$ & $52,3 \mathrm{Ba}$ & $52,4 \mathrm{~B}$ \\
\hline $60-100$ & $50,3 \mathrm{Aa}$ & $50,0 \mathrm{Ba}$ & $53,0 \mathrm{Ba}$ & $51,1 \mathrm{~B}$ \\
\hline Média & $51,1 \mathrm{a}$ & $54,4 \mathrm{a}$ & 55,9 a & - \\
\hline
\end{tabular}

Médias seguidas de mesma letra não diferem entre si pelo teste de Tukey (p<0,05), e as letras minúsculas comparam os manejos dentro da mesma profundidade e as maiúsculas, as profundidades dentro de cada tipo de manejo.

Means followed by the same letters did not differ from one another by the Tukey test $(p<0.05)$. Lower case letters compare the types of management within the same depth and capital letters compare the depths within each type of management. 
Tabela 4 - Teores foliares de macro e micronutrientes da seringueira, em função dos manejos mecânicos (grade e roçadeira) e cultura de cobertura (Pueraria) na entrelinha da seringueira, após oito anos de cultivo. Jaboticabal, SP

Table 4-Rubber tree macro and micronutrient foliar contents as a function of mechanical management (grazer and harrow) and cover crop (Pueraria), in inter rows of rubber trees after eight years of culture. Jaboticabal, SP

\begin{tabular}{|c|c|c|c|c|}
\hline \multicolumn{5}{|c|}{$\mathrm{N}\left(\mathrm{g} \mathrm{kg}^{-1}\right)$} \\
\hline Clones & Grade & Roçadora & Pueraria & Médias \\
\hline RRRIM 701 & $25,6 \mathrm{Ab}$ & $27,3 \mathrm{Aa}$ & $26,5 \mathrm{Bab}$ & $26,5 \mathrm{~B}$ \\
\hline PB 235 & $27,6 \mathrm{Aa}$ & $26,3 \mathrm{Aa}$ & $27,9 \mathrm{Aa}$ & $27,2 \mathrm{~A}$ \\
\hline Média & $26,6 \mathrm{a}$ & $26,8 \mathrm{a}$ & $27,20 \mathrm{a}$ & - \\
\hline \multicolumn{5}{|c|}{$\mathrm{P}\left(\mathrm{g} \mathrm{kg}^{-1}\right)$} \\
\hline RRRIM 701 & $1,8 \mathrm{Bb}$ & $2,2 \mathrm{Ba}$ & $1,9 \mathrm{Bab}$ & $2,0 \mathrm{~B}$ \\
\hline PB 235 & $2,1 \mathrm{Ab}$ & $2,1 \mathrm{Aa}$ & $2,2 \mathrm{Aab}$ & $2,1 \mathrm{~A}$ \\
\hline & $1,9 \mathrm{~b}$ & $2,2 \mathrm{a}$ & $2,1 \mathrm{ab}$ & - \\
\hline \multicolumn{5}{|c|}{$\mathrm{K}\left(\mathrm{g} \mathrm{kg}^{-1}\right)$} \\
\hline RRRIM 701 & 11,6 & 11,3 & 10,6 & $11,2 \mathrm{~A}$ \\
\hline PB 235 & 12,1 & 11,6 & 11,5 & $11,7 \mathrm{~A}$ \\
\hline Média & $11,9 \mathrm{a}$ & $11,4 \mathrm{a}$ & $11,0 \mathrm{ab}$ & - \\
\hline \multicolumn{5}{|c|}{$\mathrm{Ca}\left(\mathrm{g} \mathrm{kg}^{-1}\right)$} \\
\hline RRRIM 701 & $7,2 \mathrm{Aab}$ & $8,9 \mathrm{Aa}$ & $7,7 \mathrm{Ab}$ & $7,9 \mathrm{~A}$ \\
\hline PB 235 & $9,0 \mathrm{Aab}$ & $9,7 \mathrm{Aa}$ & $7,8 \mathrm{Ab}$ & $8,8 \mathrm{~A}$ \\
\hline Média & $8,1 \mathrm{ab}$ & $9,3 \mathrm{a}$ & $7,7 \mathrm{~b}$ & - \\
\hline \multicolumn{5}{|c|}{$\mathrm{Mg}\left(\mathrm{g} \mathrm{kg}^{-1}\right)$} \\
\hline RRRIM 701 & $4,4 \mathrm{Aa}$ & $4,8 \mathrm{Aa}$ & $4,5 \mathrm{Aa}$ & $4,5 \mathrm{~A}$ \\
\hline PB 235 & $4,3 \mathrm{Ba}$ & $4,1 \mathrm{Ba}$ & $3,7 \mathrm{Ba}$ & $4,0 \mathrm{~B}$ \\
\hline Média & $4,3 \mathrm{a}$ & $4,5 \mathrm{a}$ & $4,1 \mathrm{a}$ & \\
\hline \multicolumn{5}{|c|}{$\mathrm{B}\left(\mathrm{mg} \mathrm{kg}^{-1}\right)$} \\
\hline$\overline{\text { RRRIM } 701}$ & $30,8 \mathrm{Aa}$ & $28,5 \mathrm{Aa}$ & $28,8 \mathrm{Aa}$ & $29,3 \mathrm{~A}$ \\
\hline PB 235 & $27,8 \mathrm{Ba}$ & $23,8 \mathrm{Ba}$ & $24,8 \mathrm{Ba}$ & $25,4 \mathrm{~B}$ \\
\hline Média & $29,3 a$ & $26,1 \mathrm{a}$ & $26,8 \mathrm{a}$ & \\
\hline
\end{tabular}

Avaliando os manejos na nutrição da seringueira, notou-se que houve interação do nitrogênio; o clone RRIM701 apresentou o maior teor desse nutriente no manejo com roçadora e Pueraria, ao passo que no clone PB235 os manejos foram semelhantes com relação a esse nutriente. No caso da Pueraria, era de se esperar maior concentração de $\mathrm{N}$ foliar, com a sua decomposição no solo (mineralização), visto que na literatura é amplamente conhecida a capacidade de essa leguminosa fixar $\mathrm{N}$ atmosférico, aumentando o teor de $\mathrm{N}-\mathrm{NO}_{3}^{-}$do solo (HEINZMANN, 1985). Entretanto, deve-se considerar que o adubo verde muitas vezes não tem capacidade para suprir suficientemente as necessidades das culturas, havendo a necessidade de uso associado aos fertilizantes minerais (FRYLE et al., 1985).
Para o teor de fósforo e cálcio, observou-se maior valor no tratamento com roçadora, em ambos os clones (Tabela 4). Diante desses resultados, notou-se que as espécies espontâneas manejadas com roçadora foram semelhantes à Pueraria na reciclagem de nutrientes (N, P e Ca) absorvidos pela seringueira. Nesse sentido, Favero et al. (2000) avaliaram plantas daninhas e leguminosas, comprovando o potencial das primeiras na ciclagem de nutrientes ( $\mathrm{P}, \mathrm{K}$ e $\mathrm{Mg}$ ). Tais resultados podem ser explicados também pela semelhança desses manejos na fertilidade do solo, a partir dos valores da soma de bases e da saturação por bases (Tabela 3 ).

Os teores foliares de nutrientes na seringueira, em função dos manejos, variaram de 26,6 a $27,2 \mathrm{~g} \mathrm{~kg}^{-1}$ para o $\mathrm{N}$ e estão abaixo do valor considerado adequado (33-37 $\mathrm{g} \mathrm{kg}^{-1}$ ); o fósforo apenas no manejo com grade 
apresentou teor abaixo do considerado adequado (2$\left.2,5 \mathrm{~g} \mathrm{~kg}^{-1}\right)$. Os demais nutrientes como cálcio, magnésio e boro exibiram, em todos os manejos, teores acima do considerado adequado (PUSHPARAJAH, 1992).

Notou-se, ainda, que os maiores teores foliares de $\mathrm{N}$ e $\mathrm{P}$ foram obtidos no clone PB235, ao passo que no clone RRIM701 os maiores teores foram constatados para os nutrientes Mg e B (Tabela 4). Portanto, podese inferir que o clone PB235 é mais exigente em N e $\mathrm{P}$ e o clone RRIM701, em Mg e B. Ao passo que para o K ambos os clones foram semelhantes.

Cabe salientar que os tratamentos não afetaram significativamente os teores foliares do S (1,6 a 1,9 $\left.\mathrm{g} \mathrm{kg}^{-1}\right), \mathrm{Zn}\left(15\right.$ a $\left.25 \mathrm{mg} \mathrm{kg}^{-1}\right), \mathrm{Cu}$ (7 a $\left.10 \mathrm{mg} \mathrm{kg}^{-1}\right), \mathrm{Fe}$ (176 a $299 \mathrm{mg} \mathrm{kg}^{-1}$ ) e Mn (126 a $205 \mathrm{mg} \mathrm{kg}^{-1}$ ) (dados não apresentados).

\subsection{Efeito dos tratamentos no desenvolvimento da seringueira}

Pelos resultados foi observado que os clones de seringueira tiveram comportamento diferenciado, com os manejos afetando o clone PB. Já o clone RRIM mostrou-se indiferente aos manejos utilizados (Figura 1). Por esse resultado, pode-se inferir que os clones de seringueira respondem diferentemente ao manejo da entrelinha utilizado, admitindo-se, assim, que cada clone exige um manejo específico da entrelinha para melhor crescimento. Pushparajah (1983) admitiu a interação genótipo $\mathrm{x}$ ambiente em seringueira, visto que os fatores ligados ao solo e ao clima afetam componentes vegetativos dos clones.

Cabe salientar ainda que, independentemente do manejo, o clone PB235 apresentou maior desenvolvimento, comparado ao clone RRIM. Esse maior vigor do clone PB235 também foi verificado em estudos de competição com vários clones no Estado de São Paulo por Gonçalves et al. (1993).

Notou-se no clone PB que o uso da grade e da roçadora foi significativamente superior à Pueraria, enquanto para o clone RRIM não houve diferença significativa. Considerando, porém, a média dos dois clones, observou-se que a Pueraria foi o pior tratamento para crescimento da seringueira. Resultados semelhantes foram obtidos por Martins (2000), em seringueira em formação cultivada no Estado de São Paulo, onde o autor verificou que o manejo da entrelinha com plantas de cobertura (centrosema, kudzu, guatá, soja perene e galáxia), comparado à roçadora, apresentou decréscimo no desenvolvimento da seringueira, especialmente a partir do terceiro ano.

Os efeitos da Pueraria não foram expressivos, visto que sua ação ficou restrita à camada superficial do solo, conforme pode ser visto nas alterações sobre a fertilidade (Tabela 3); isso foi devido, possivelmente, ao desenvolvimento do sistema radicular restrito em profundidade, minimizando a ciclagem de nutrientes. O fato de que o cultivo intercalar de Pueraria, sem adubação complementar, pode ter concorrido, em determinadas fases de desenvolvimento, com a seringueira por nutrientes, fato relatado na literatura com outras culturas perenes (VAN HUYSSTEEN e WEBER, 1980). Em seringueira, Chandapillai (1968) atribui a esse fato a alta concentração de raízes das plantas de cobertura (kudzu tropical e centrosema). A hipótese da concorrência da Pueraria com a seringueira por nutrientes ficou enfraquecida neste trabalho, visto que a cultura de cobertura não diminuiu a fertilidade do solo (Tabela 3 ), em face dos outros manejos adotados.

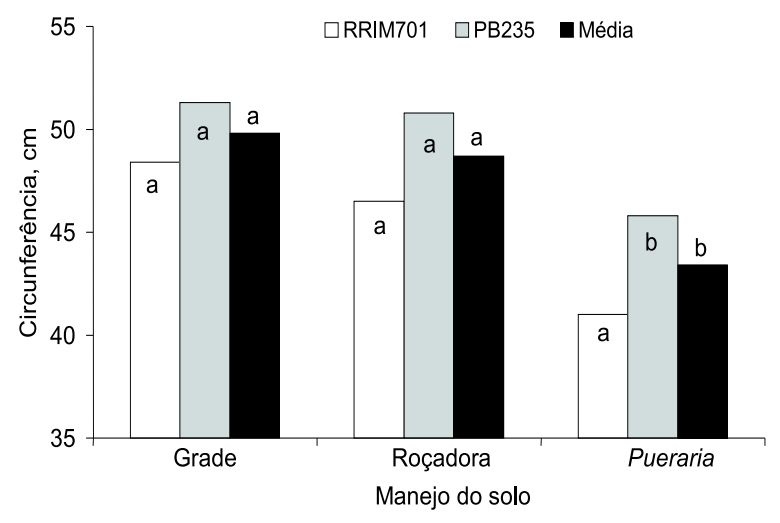

Figura 1 - Circunferência à altura do peito em função dos manejos mecânicos (grade e roçadora) e com cultura de cobertura (Pueraria) na entrelinha da seringueira, clones RRIM701 e PB235, após oito anos de cultivo. Jaboticabal, SP. Médias seguidas de mesma letra não diferem entre si, pelo teste de Tukey $(\mathrm{p}<0,05)$ os diferentes clones.

Figure 1-Rubber tree diameter at breast height as a function of mechanical management (grazer and harrow) and cover crop (Pueraria) between rows of the rubber tree clones RRIM701 and PB235 after eight years of culture, Jaboticabal, SP. Means followed by the same letters did not differ by the Tukey test $(p<0.05)$.

R. Árvore, Viçosa-MG, v.29, n.2, p.185-193, 2005 


\section{CONCLUSÕES}

O manejo da entrelinha da seringueira com roçadora mostrou-se importante no sistema solo-planta. No solo, a ação do manejo da entrelinha restringiu-se à camada de $0-20 \mathrm{~cm}$ de profundidade. A influência do manejo da entrelinha da seringueira na sua nutrição depende do clone utilizado no sistema de produção.

\section{AGRADECIMENTOS}

À FAPESP, pelo apoio financeiro ao Projeto (Processo 97/07140-8).

\section{REFERÊNCIAS BIBLIOGRÁFICAS}

ANDRIOLI, I.; CENTURION, J.F. Levantamento detalhado dos solos da Faculdade de Ciências Agrárias e Veterinárias de Jaboticabal. In: CONGRESSO BRASILEIRO DE CIÊNCIA DO SOLO, 27., Brasília, 1999. Anais... Brasília: Sociedade Brasileira de Ciência do Solo, 1999. p.1-4.

BATAGLiA, O.C. et al. Métodos de análise química de plantas. Campinas: Instituto Agronômico de Campinas, 1983. 48p. (Boletim Técnico, 78).

BAYER, C.; MIELNICZUK, J.; MARTIN-NETO, L. Efeito de sistemas de preparo e de cultura na dinâmica da matéria orgânica e na mitigação das emissões de $\mathrm{CO}_{2}$. Revista Brasileira de Ciência do Solo, v.24, p.599-607, 2000.

CACERES, N.T. Adubação verde com leguminosas em rotação com cana-deaçúcar (Saccharum spp.). 1994. 45f. Dissertação (Mestrado em Agronomia) - Escola Superior de Agricultura Luiz de Queiroz, Piracicaba, 1994.

CARDOSO, M. Instruções para a cultura da seringueira. Campinas: Instituto Agronômico de Campinas, 1989.50p. (Boletim, 196).

CASTRO FILHO, C.; VIEIRA, M.J.; CASÃO JÚNIOR, R. Tillage methods and soil and water conservation in Southern Brazil. Soil Tillage Research, v.20, p.271-283, 1991.

CENTURION, J. F.; CENTURION, M. A. P. C.; ANDRIOLI, I. Rubber growing soils of São Paulo, Brazil. Indian Journal Natural Rubber Research, v.8, n.2, p.75-84, 1995.

R. Árvore, Viçosa-MG, v.29, n.2, p.185-193, 2005
CHANDAPILLAI, M.M. Studies of root systems of some cover plants. Journal of the Rubber Research Institute of Malaya, v.20, n.3, p.117-129, 1968.

CHAPMAN, H.D. Técnicas de amostragem para o diagnóstico foliar: árvores frutíferas e pequenos frutos. Fertilié, v.27, p.75-91,1966.

COMPAGNON, P. Fertilization In: COMPAGNON, P. (Ed.). Le caoutchouc naturel. Paris: Maisonneuve et Larose, 1986.p.297-314.

CORTEZ, J.V. A evolução da cultura da seringueira no estado de São Paulo-Brasil. In: CICLO DE PALESTRAS SOBRE A HEVEICULTURA

PAULISTA, 1., 1998. Barretos. Anais... Barretos: SSA/APABOR, 1998. p.61-92.

FAVERO, C. et al. Crescimento e acúmulo de nutrientes por plantas espontâneas e por leguminosas utilizadas para adubação verde. Revista Brasileira de Ciência do Solo, v.24, p.171-177, 2000.

FRYLE, W.W.; SMITH, W.G.; WILLIAMS, R.J. Economics of winter cover crops as a source of nitrogen for no-till corn. Journal Soil Water Conservation, v.40, p.246-249, 1985.

GONÇALVES, P.S. et al. Desempenho preliminar de clones de seringueira na região de São José do Rio Preto, planalto do Estado de São Paulo. Bragantia,v.52,n.2,p.119-130,1993.

HAYNES, R.J. The decomposition process mineralization, humus formation, and degradation. In: HAYNES, R.J., (Ed.) Mineral nitrogen in the plant-soil systems. Orlando, Academic Press, 1986. p.52-126. (Physiological Ecology. A. Series of Monographs, Texts, and Treatises).

HEINZMANN, F.X. Resíduos culturais de inverno e assimilação de nitrogênio por culturas de verão. Pesquisa Agropecuária Brasileira, v.20, n.9, p.1021-1030, 1985.

HOYT, P.B. Effects of organic matter content on exchangeable $\mathrm{Al}$ and $\mathrm{pH}$-dependent acidity of very acid soils. Canadian Journal Soil Science, v.57,p.221-222,1977. 
MARTINS, A.L.M. Formação da cultura da seringueira [Hevea brasilienses (willd. Ex Adr. De Juss.) Muell-Arg.] através do uso de leguminosas perenes. 2000, 83f. Tese (Doutorado em Produção Vegetal). Universidade Estadual de São Paulo, Jaboticabal, 2000 .

ORTOLANI, A.A. et al. Aptidão agroclimática para regionalização da heveicultura no Brasil. In: SEMINÁRIO NACIONAL SOBRE RECOMENDAÇÃO DE CLONES DDE SERINGUEIRA, 1.,1983, Brasília. Anais... Brasília: EMBRAPA/SUDHEVEA, 1983. p.19-28.

PIMENTAL-GOMES, F. Curso de estatística experimental. 11.ed. São Paulo: Nobel, 1985. 466p.

PRADO, R.M.; CENTURION, J.F. Alterações na cor e no grau de floculação de um Latossolo Vermelho-Escuro sob cultivo contínuo de cana-deaçúcar. Pesquisa Agropecuária

Brasileira, v.36, p.197-203, 2001.

PUSHPARAJAH, E. Problems and potentials for establishing Hevea under difficult environmental conditions. Planter, v.50, p.242-251, 1983.

PUSHPARAJAH, E. Rubber. In: WICHMANN, W. (Ed.). World fertilizer use manual. Paris: International Fertilizers Industry Association, 1992.p.491-498.

RAIJ, B. van., QUAGGIO, J. A. Métodos de análise de solo para fins de

fertilidade. Campinas: Instituto Agronômico de Campinas, 1983. 31p. (Boletim Técnico, 81).
RAIJ, B.van. et al. (Eds.) Análise química para avaliação da fertilidade do solo. Campinas: Instituto Agronômico de Campinas, 2001. 285p.

\section{SAS INSTITUTE. SAS/STAT procedure guide for personal computers. 5 ed. Cary: 1996.}

SERINGUEIRA. Crise aperta e preços mundiais despencam. In: NEHMI, I.M.D. et al. (Eds.) AGRIANUAL 99: anuário da agricultura brasileira. São Paulo: FNP, 1999.p.438-447.

SOUZA, M.M.S.R. Ação da cobertura com leguminosas sobre o solo e o seringal em formação. 1996. 88f. Dissertação (Mestrado em Agronomia) - Escola Superior de Agricultura “Luiz de Queiroz", Piracicaba, 1996.

TESTA, V.M.; TEIXEIRA, L.A.J.; MIELNICZUK, J. Características químicas de um Podzólico Vermelho-Escuro afetadas por sistemas de culturas. Revista Brasileira de Ciência do Solo, v.16, n.1, p.107-114,1992.

TIWARI, K.N.; TIWARI, S.P.; PATHAK, A.N. Studies on green manuring of rice in double cropping system in a partially reclained saline sodic soil. Indian Jornal of Agronomy, v.25, n.1, p.136-145, 1980.

VAN HUYSSTEEN, L.; WEBER, H.W. The effect of selected minimum and conventional tillage practices in vineyard cultivation on vine performace. South African Journal for Enology and Viticulture, v.1, n.2, p.77-83, 1980. 